\title{
Norois
}

Environnement, aménagement, société

$216 \mid 2010 / 3$

Territoire, Paysage, Anthropisation, Perception,

Conservation, Restauration

\section{La conservation d'une espèce patrimoniale en déclin au sein d'un agrosystème. Le cas de la Guifette noire Chlidonias niger (Linnaeus, 1758) dans le Marais poitevin}

The conservation of a patrimonial and declining species in an agrosystem. The case of the Black tern Chlidonias niger (Linnaeus, 1758) in the Marais poitevin

\section{Alain Thomas et Laurent Godet}

\section{(2) OpenEdition}

\section{Édition électronique}

URL : https://journals.openedition.org/norois/3307

DOI : $10.4000 /$ norois.3307

ISBN : 978-2-7535-1564-2

ISSN : $1760-8546$

\section{Éditeur}

Presses universitaires de Rennes

Édition imprimée

Date de publication : 15 novembre 2010

Pagination : 7-23

ISBN : 978-2-7535-1257-3

ISSN : 0029-182X

\section{Référence électronique}

Alain Thomas et Laurent Godet, «La conservation d'une espèce patrimoniale en déclin au sein d'un agrosystème. Le cas de la Guifette noire Chlidonias niger (Linnaeus, 1758) dans le Marais poitevin », Norois [En ligne], 216 | 2010/3, mis en ligne le 01 décembre 2012, consulté le 13 janvier 2022. URL : http://journals.openedition.org/norois/3307 ; DOI : https://doi.org/10.4000/norois.3307 


\title{
LA CONSERVATION D'UNE ESPÈCE PATRIMONIALE EN DÉCLIN AU SEIN D'Un agrosystème. Le CAS de la Guifette noire Chlidonias Niger (Linnaeus, i758) Dans le Marais poitevin
}

\author{
Alain Thomas \\ 11, rue Marcel-Lebois, Moricq -85750 AngLes - France \\ alain.thomas90@orange.fr \\ LAurent Godet \\ laboratoire GÉOLITTOMER - LETG UMR 6554 CNRS \\ (Université de Nantes), \\ chemin de la Censive du Tertre, BP 81227 - 44312 NANTES cedex 3 - France \\ godet@mnhn.fr
}

\section{RÉSUMÉ}

Le Marais poitevin, deuxième zone humide de France, a été progressivement mis en culture au point de devenir un agrosystème. Les prairies humides (pour l'essentiel pâturées), considérées comme des vestiges de l'ancienne zone humide, sont les espaces sur lesquels se concentrent les mesures de conservation de la biodiversité du marais. Parmi les espèces patrimoniales associées à ces milieux, nous présentons l'exemple de la Guifette noire Chlidonias niger (Linnaeus, 1758). Cette espèce menacée a été à l'origine de multiples mesures de conservation (aires protégées et aménagements lui étant spécialement consacrés) dans le marais. Cette étude révèle que de telles mesures, bien qu'ayant des effets positifs, ne parviennent toutefois pas à enrayer le déclin significatif de la population qui se concentre de plus en plus sur les espaces bénéficiant d'une gestion environnementale. L'exemple de cette espèce de zone humide, aujourd'hui présente au sein de milieux de substitution qu'offre un agrosystème, pose la question des choix de politiques conservatoires qui doivent être opérés dans un contexte de changement d'occupation du sol à large échelle. Doit-on encore développer des mesures de gestion interventionniste ou privilégier la restauration de la fonctionnalité de milieux pour conserver des espèces menacées?

MotS CLÉ : Guifette noire - Chlidonias niger - Marais poitevin - prairie pâturée conservation

\section{ABSTRACT}

The conservation of a patrimonial and declining species in an agrosystem. The case of the Black tern Chlidonias niger (Linnaeus, 1758) in the Marais poitevin

The Marais poitevin, the second largest wetland in France, has been progressively cultivated, and can be considered as an agrosystem. Humid grasslands (essentially submitted to cattle grazing), are considered as the last remaining wetlands of the area, and biodiversity conservation measures are now focusing on them. Among the patrimonial 
grassland species, we interested in the Black tern Chlidonias niger (Linnaeus, 1758). Several conservation measures (protected areas, environmental management) have been deployed in the area to conserve this threatened species. However, despite some positive effects of these measures, the species is still significantly declining and is now concentrating in areas benefiting from an environmental management. The example of such a wetland species living in an agrosystem raises the question of the conservation policies to adopt in the current context of large-scale land-use changes. Should we develop interventionist management measures or preferring the restoration of habitat functionalities to conserve threatened species?

KEY WORDS : Black tern-Chlidonias niger - Marais poitevin-pasture-conservation

Les zones humides sont connues pour être des écosystèmes parmi les plus diversifiés du monde (Keddy, 2000) et parmi ceux rendant le plus de services écologiques aux sociétés humaines (MEA, 2005). En Europe comme aux États-Unis, leur perte a été estimée à plus de $50 \%$ en 200 ans (Finlayson et Davidson, 1999; Dahl, 2000) et presque toutes sont aujourd'hui soumises à des dégradations d'origine anthropique, pour une grande part liées à l'agriculture (Brinson et Malvarez, 2002). En réponse à ces dégradations, la conservation de ces espaces a fait l'objet de plusieurs mesures plus ou moins coercitives de conservation (réseau actuel de 1674 sites RAMSAR à travers le monde totalisant 150 millions d'hectares (Keddy et al., 2009) par exemple) et mesures ponctuelles de gestion destinées aux espèces les plus menacées qui leur sont associées.

Le Marais poitevin, deuxième zone humide française couvrant environ 100000 ha, peut être considéré comme un exemple représentatif de l'état de conservation des zones humides européennes. Très tôt mis en valeur pour l'agriculture, les zones humides y ont très vite été remplacées par des cultures. Les prairies, pour l'essentiel pâturées, considérées comme des vestiges des anciennes zones humides du marais ont régressé de $50 \%$ de 1973 à 2006 pour couvrir aujourd'hui environ 28000 ha de prairies permanentes (Parc Interrégional du Marais Poitevin (PIMP), données non publiées). Jusqu'à présent, la mise en place de mesures de gestion conservatoire a été la voie privilégiée pour conserver la biodiversité de ces milieux, à travers deux voies :

- gestion conservatoire centrée sur les milieux, via la création d'espaces protégés bénéficiant d'une gestion environnementale (Réserves Naturelles, acquisitions foncières à gestion environnementale, etc.) qui couvrent actuellement 7200 ha de prairies;

- gestion conservatoire " espèce-centrée », via des aménagements mis en place pour l'accueil d'espèces bien particulières.

Parmi les espèces associées aux prairies humides du marais, nous présentons ici l'exemple de la Guifette noire Chlidonias niger. Cette espèce, inscrite à l'Annexe I de la Directive Oiseaux, considérée comme rare et en déclin à l'échelle de l'Europe de l'Ouest (Tucker et al., 1994; Beintema, 1997), fait aujourd'hui l'objet de nombreuses mesures de conservation. En France, les colonies sont cantonnées à cinq zones humides du Grand Ouest (complexe des Marais du Brivet et de Brière, Lac de Grand Lieu, Marais poitevin, Marais de Rochefort et Brenne), avec un nombre de couples oscillant de 94 à 415 sur la période 1992-2008 et un total de 213 recensés en 2008 (Trotignon, 2009). Le Marais poitevin accueille $10 \%$ de la population nationale de Guifettes noires, qui se concentrent dans cette région sur les milieux de prairies humides pâturées. Lors de la nidification, les couples de cette espèce s'installent sur les dépressions les plus humides, appelées «baisses ».

Aujourd'hui, l'espèce y est considérée comme un des oiseaux à la fois menacés et emblématiques de ces prairies. Le PIMP soutient financièrement le suivi de l'espèce sur l'ensemble du marais. Une action «Guifette noire » a été conduite dans le cadre du programme Life «Conservation 
des habitats et des espèces les plus remarquables du Marais poitevin » sur la période 2004-2008. En outre, il s'agit d'une des espèces du cortège d'oiseaux nichant en prairie humide qui est à l'origine de la création de plusieurs espaces protégés, telle que la Réserve Naturelle Nationale de Saint-Denis-du-Payré et de terrains acquis par la Ligue pour la Protection des Oiseaux (LPO). Par ailleurs, différents dispositifs " espèce-centrée » sont mis en place pour favoriser sa reproduction, tels que des « radeaux-nichoirs », favorisant la construction de nids sur des plans d'eau, ou encore des clôtures protégeant les nids du piétinement par le bétail.

Mais quelle est la pertinence de ces mesures de gestion conservatoire au sein d'un éco-complexe devenu aujourd'hui un agrosystème? Nous proposons d'abord une analyse de l'évolution de la population à l'échelle du Marais poitevin (effectifs et répartition). Nous évaluons ensuite l'effet des mesures de gestion centrées sur les milieux (en distinguant trois types d'espaces naturels à gestion conservatoire) et sur l'espèce (pose de radeaux-nichoirs et mesures d'exclusion du bétail) sur la population. Nous discuterons enfin des choix qui semblent aujourd'hui les plus appropriés pour conserver une telle population : doit-on poursuivre (ou augmenter) les mesures de gestion conservatoire ou privilégier la restauration de la fonctionnalité d'habitats de cette zone humide?

\section{Matériel et méthodes}

\section{Suivi de LA POPULATION : DONNÉES HISTORIQUES ET PROTOCOLE DE SUIVI RÉCENT}

Les données exploitées dans cette étude s'étalent du début du xx siècle à 2008 et sont de nature diverse et de qualité inégale. L'Annexe 1 synthétise les données exploitées. D’une manière générale, parmi l'ensemble de ces données, trois périodes peuvent être distinguées en fonction de leur précision et de leur qualité.

Jusqu'à 1975, les informations au sujet de l'espèce sont lacunaires. Durant la première moitié du xxe siècle, C. Marcot (1937) signale la nidification de l'espèce dans certains secteurs et M. Bardin (1938) signale des prélèvements d'œufs sur certains prés communaux. S'ajoutent à ces données 13 mentions de 1907 à 1939, se référant principalement à des prélèvements d'œufs, de poussins ou d'adultes à des fins de collection, auxquelles s'ajoutent au moins six mentions de nidifications possibles. Peu ou pas d'informations sont données quant aux effectifs ou localisations précises des colonies et rien n'est dit du nombre de poussins. Durant les années 1960, malgré une absence de suivi spécifique et exhaustif, il existe plusieurs données avec les premières évaluations d'effectifs de couples de certaines colonies.

La période allant de 1977 à 1991 apporte les premières évaluations quantitatives systématiques du nombre de couples des colonies mentionnées, ainsi que leur localisation, même si le suivi n'est pas exhaustif à l'échelle du Marais poitevin. Aucune donnée du nombre de poussins n'est en revanche fournie.

Il faut attendre 1992 pour voir débuter un travail de suivi de l'espèce quasi-exhaustif à l'échelle du marais (nombre et localisation des colonies, nombre de couples, nombre de poussins menés à l'envol). À partir de 1993, le suivi peut être considéré comme fiable (à \pm 5 couples). Toutes les analyses statistiques réalisées dans le présent article ne traitent que des données s'étalant sur cette période 1992-2008. À partir de 1992, la population a été suivie par plusieurs naturalistes dont l'un des deux auteurs du présent article (AT) en est le principal. Le protocole de suivi a été le même de 1992 à 2008 et s'articule en six phases principales correspondant à la phénologie de l'espèce dans le Marais poitevin (tableau 1). 
Alain Thomas, Laurent Godet

\begin{tabular}{|c|c|c|}
\hline $\begin{array}{l}\text { Périodes indi- } \\
\text { catives }\end{array}$ & $\begin{array}{l}\text { Phénologie de } \\
\text { l'espèce }\end{array}$ & Étapes du protocole \\
\hline $1^{\mathrm{er}}-20$ mars & $\begin{array}{l}\text { Oiseaux sur les } \\
\text { sites d'hivernage } \\
\text { africains }\end{array}$ & $\begin{array}{l}\text { Tous les sites favorables ou ayant déjà accueilli des couples les années passées } \\
\text { sont visités afin de repérer ceux qui sont potentiellement les plus attractifs. }\end{array}$ \\
\hline 20 mars-20 avril & $\begin{array}{l}\text { Arrivée prénup- } \\
\text { tiale }\end{array}$ & $\begin{array}{l}\text { Les adultes de retour de leurs quartiers d'hivernage africains se rassemblent sur } \\
\text { des « sites bases » ( } 3 \text { sites principaux). À partir du 20-25 mars ces sites sont visi- } \\
\text { tés régulièrement afin de dénombrer et d'analyser le comportement des oiseaux } \\
\text { ( } 3 \text { à } 7 \text { visites hebdomadaires). }\end{array}$ \\
\hline 20 avril- 15 mai & $\begin{array}{l}\text { Prospection des } \\
\text { sites de nidifica- } \\
\text { tion potentiels }\end{array}$ & $\begin{array}{l}\text { Dès la fin du mois d'avril jusqu'à la mi-mai, la pression d'observation augmente } \\
\text { encore : tous les sites potentiellement attractifs sont régulièrement visités et, } \\
\text { pour chaque oiseau contacté, sa localisation et sa trajectoire sont systématique- } \\
\text { ment notées. }\end{array}$ \\
\hline 15 mai-30 mai & $\begin{array}{l}\text { Installation des } \\
\text { couples }\end{array}$ & $\begin{array}{l}\text { Dès la mi-mai, lorsque les couples commencent à être cantonnés sur des prairies, } \\
\text { un plan panoramique de terrain est réalisé pour chaque colonie en notant tous les } \\
\text { éléments remarquables fixes. Tout le suivi est ensuite effectué depuis le même } \\
\text { point d'observation. Les nids sont repérés grâce au comportement des oiseaux } \\
\text { (poses répétées d'un couple, apports/offrandes de nourriture, décollage d'indivi- } \\
\text { dus à l'approche d'un rapace etc.). Un numéro est attribué à chaque nid qui est } \\
\text { localisé le plus précisément sur le plan panoramique. Pour le premier repérage } \\
\text { des nids, } 2 \text { heures d'observation sont nécessaires. Suivant la configuration du site } \\
\text { et l'effectif, cette durée peut être supérieure. }\end{array}$ \\
\hline 30 mai-20 juin & $\begin{array}{l}\text { Nidification des } \\
\text { couples }\end{array}$ & $\begin{array}{l}\text { Une fois les nids numérotés et localisés, } 1 \text { heure (durée minimale) de suivi tous } \\
\text { les } 3 \text { jours est réalisée lors de la période d'incubation jusqu'à l'éclosion et les } \\
\text { premiers envols des jeunes. }\end{array}$ \\
\hline 20 juin-20 juillet & $\begin{array}{l}\text { Dispersion des } \\
\text { individus }\end{array}$ & $\begin{array}{l}\text { Lors de la période d'envol des poussins, un passage quasi quotidien est effectué } \\
\text { pour les dénombrer. Ce suivi est réalisé sur les sites d'implantation des colonies, } \\
\text { mais également sur les sites « bases ", certains jeunes quittant les colonies pour } \\
\text { s'y rassembler peu après leur envol. Les dates d'envol et la durée de stationne- } \\
\text { ment des oiseaux sur les sites de nidification sont extrêmement variables selon } \\
\text { les sites et les années. En règle générale, l'envol des jeunes a lieu entre le } 25 \text { juin } \\
\text { et le } 10 \text { juillet. }\end{array}$ \\
\hline
\end{tabular}

Tableau 1 : Protocole de suivi de la nidification de la Guifette noire dans le Marais poitevin depuis 1992 Breeding monitoring method of the Black tern in the Marais poitevin since 1992

\section{ÉVOLUTION QUANTITATIVE DE LA POPULATION}

Les données d'évolution des trois paramètres populationnels du nombre de couples, de poussins menés à l'envol et du succès moyen de reproduction (ex. nombre moyen de poussins par couple) ont été synthétisées de 1960 à 2008. Celles de 1960 à 1991 sont présentées à titre indicatif. Celles 1992 à 2008 ont fait l'objet d'une estimation de tendance d'évolution via l'utilisation de modèles de régression linéaire simple dans le cas d'une distribution normale des résidus (données éventuellement log transformées) et de Modèles Linéaires Généralisés (GLM) considérant que les données suivent une distribution de Poisson dans le cas contraire.

\section{ÉVOLUTION SPATIALE DE LA POPULATION}

A l'échelle du Marais poitevin, toutes les données anciennes (si localisées par les auteurs) et récentes des sites d'implantation des colonies ont été cartographiées. Avant 1975, seules des données qualitatives peuvent être synthétisées sous forme de cartes. De 1977 à 2008 les données de nombre de couples des colonies ont été cartographiées. Le niveau de précision dans la localisation des colonies, est mentionné sur chaque carte selon 3 niveaux : certain (la donnée est localisée à quelques mètres de précision); probable (la donnée peut concerner deux à trois parcelles proches); 
possible (la donnée peut concerner quatre à cinq parcelles proches). Les données n'ayant qu'une précision à l'échelle de la commune n'ont pas été cartographiées.

\section{EFFETS DES MESURES DE GESTION ESPÈCE-CENTRÉE}

Certains sites où nichent les guifettes font l'objet de deux types de mesures de gestion : i) pose de «radeaux-nichoirs » sur 7 sites au moins 1 année (photo 1), ii) exclusion du bétail (pose de clôtures ou retrait temporaire du bétail pendant la période de nidification; 12 sites au moins 1 année). De 1992 à 2008, nous avons testé l'impact de ces deux mesures de gestion sur les 3 paramètres populationnels suivants : le nombre de couples s'installant sur la prairie, le nombre de poussins menés à l'envol et le succès de reproduction moyen des couples.

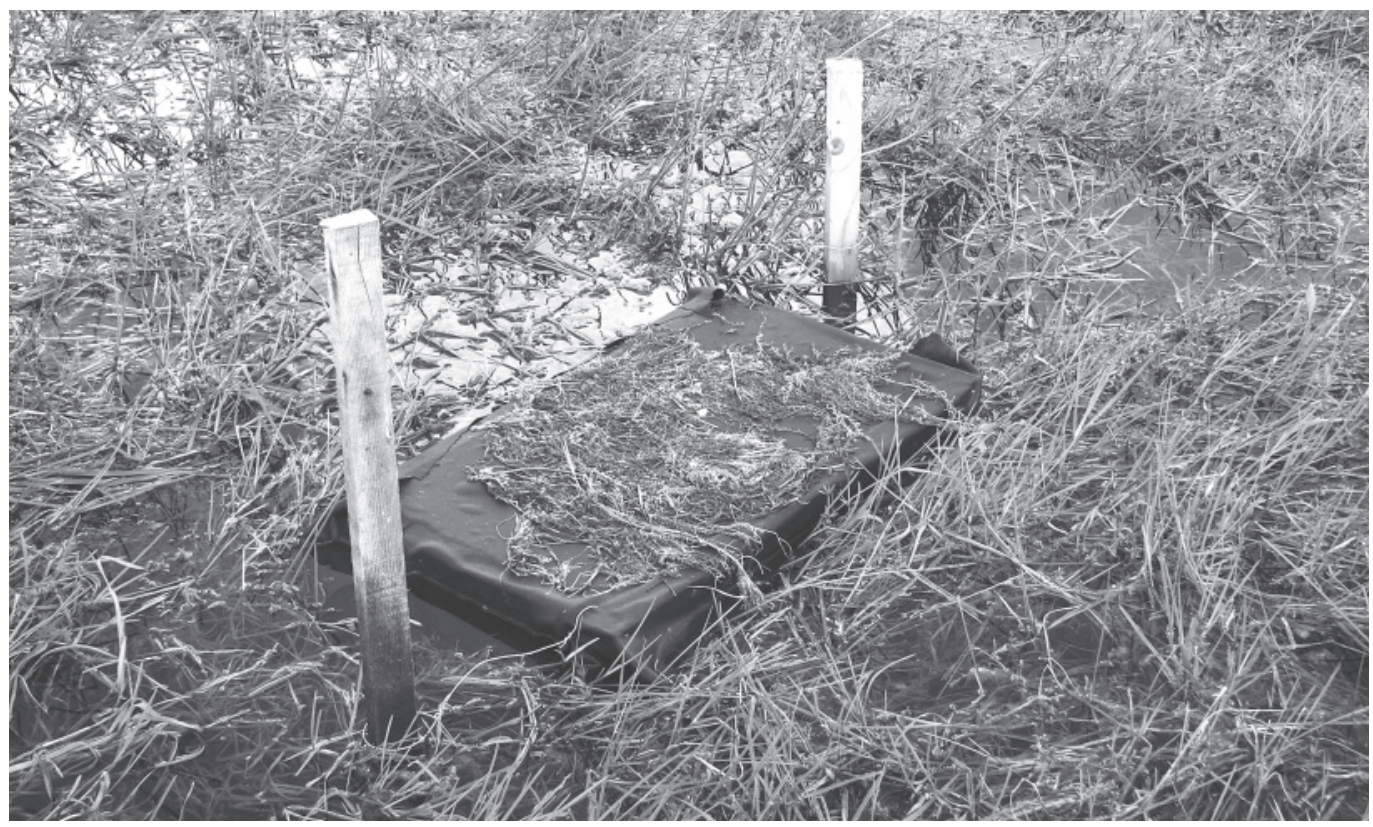

Photo 1 : Radeau-nichoir installé sur le communal des Magnils-Reigners en 2008 (Photo (C) Alain Thomas) Floating nest platform in the communal des Magnils-Reigners in 2008 (Photo (C) Alain Thomas)

Un modèle mixte d'analyse de variance a été utilisé, avec la présence de radeaux et l'exclusion du bétail considérés comme facteurs fixes, le site et l'année considérés comme facteurs aléatoires car une dépendance des paramètres populationnels en fonction de l’année et du site est suspectée. Le modèle considère que les paramètres populationnels suivent une loi de Poisson. Les analyses de variance ont été réalisées avec le logiciel $\mathrm{R}$ grâce au package lme4 (fonction lmer), permettant d'utiliser des modèles mixtes d'analyse de variance pour des variables suivant une loi de Poisson. Seuls les sites avec au moins un couple à l'année $n$ ont été pris en compte pour tester le succès moyen de reproduction.

\section{EFFETS DES MESURES DE GESTION CENTRÉE SUR LES MILIEUX}

De 1992 à 2008, nous avons testé un éventuel effet de la protection des milieux sur les 3 paramètres populationnels suivants : le nombre de couples, le nombre de poussins menés à l'envol et le succès moyen de reproduction. Trois niveaux de protection ont été considérés : 
Alain Thomas, Laurent Godet

P1 : Fort niveau de protection : Réserve Naturelle Nationale ou Régionale, terrains de la Ligue de Protection des Oiseaux (LPO).

P2 : Niveau de protection intermédiaire : prés communaux bénéficiant d'une convention de gestion environnementale.

P3 : Pas de protection.

Les différences de succès moyens de reproduction en fonction des 3 niveaux de protection ont été testées par un modèle mixte d'analyse de variance, le niveau de protection considéré comme facteur fixe et l'année comme facteur aléatoire. Le modèle considère que le succès de reproduction suit une loi de Poisson. L'analyse de variance a été réalisée avec le logiciel $\mathrm{R}$ grâce au package lme4 (fonction lmer), permettant d'utiliser les modèles mixtes d'analyse de variance pour des variables suivant une loi de Poisson. Seuls les sites avec au moins un couple à l'année $n$ ont été pris en compte.

Les nombres totaux de couples et de poussins, ne peuvent être directement comparés de 1992 à 2008 entre les 3 niveaux de protection puisque les surfaces des sites de nidification relevant de chaque type de protection diffèrent de manière importante et leurs nombres évoluent dans le temps. Nous avons d'abord calculé des «effectifs théoriques » de ces 2 paramètres populationnels (nombre de couples et de poussins) par type de protection en supposant une distribution spatiale homogène de ces 2 paramètres en fonction des surfaces disponibles pour chaque type de protection. En conséquence l'effectif théorique du nombre de couples (ou de poussins) sur les sites bénéficiant d'une protection Px est égal au nombre total de couples (ou de poussins) pondéré par la part de la superficie des sites de nidification bénéficiant d'une protection Px parmi l'ensemble des sites occupés par l'espèce de 1992 à 2008. Par exemple, pour un total de 100 couples et 20 ha de sites protégés et 80 ha non protégés, l'effectif théorique est de 20 couples sur les sites protégés, 80 couples sur les sites non protégés. Enfin, nous avons calculé un « taux de concentration par niveau de protection » $(y)$ comme suit : $y=$ (effectif observé - effectif théorique)/effectif théorique. $y$ est positif lorsqu'il y a plus de couples ou de poussins que l'effectif théorique sur les sites bénéficiant d'un niveau de protection particulier, et inversement. Les superficies des sites pris en compte pour les analyses sont celles des «baisses » (dépressions humides sur lesquelles s'installent les oiseaux) de chaque site, qui ont été évaluées par une photo-inteprétation complétée par une validation de terrain. De 1992 à 2008, les valeurs de y ont été comparées par un modèle mixte d'analyse de variance avec le niveau de protection considéré comme facteur fixe, et l'année comme facteur aléatoire. Le modèle considère que les paramètres populationnels (nombre de couples et nombre de poussins) suivent une loi de Poisson. Les analyses de variance ont été réalisées sous R grâce au package lme4 (fonction lmer), permettant d'utiliser des modèles mixtes d'analyse de variance pour des variables suivant une loi de Poisson.

Enfin, de 1992 à 2008, les tendances d'évolution du succès moyen de reproduction et des valeurs de $y$ pour le nombre de couples et de poussins selon les trois niveaux de protection sont testées via l'utilisation de modèles de régression linéaire simple dans le cas d'une distribution normale des résidus (données éventuellement log transformées) et d'un GLM considérant une distribution des données selon une loi de Poisson dans le cas contraire.

\section{Résultats}

\section{TENDANCE D'ÉVOLUTION DES EFFECTIFS}

La figure 1 présente l'évolution du nombre de couples et de poussins de Guifettes noires menés à l'envol dans le Marais poitevin de 1960 (première évaluation du nombre de couples) à 2008. Durant la période 1960-1991, avec un suivi non exhaustif de la population, des effectifs de couples jamais égalés depuis 1992 sont notés, avec notamment 60 couples en 1960, 83 en 1968, 70 en 1969 et 47 en 1982. Durant la période 1992-2008 une tendance significative à la baisse du nombre de couples est notée (modèle de régression linéaire : $\mathrm{R}^{2}=0,51$; $\mathrm{p}=0,001$ ). La taille moyenne 
Figure 1 : Nombre de couples et de poussins de 1960 à 2008

Number of breeding pairs and chicks from 1960 to 1980

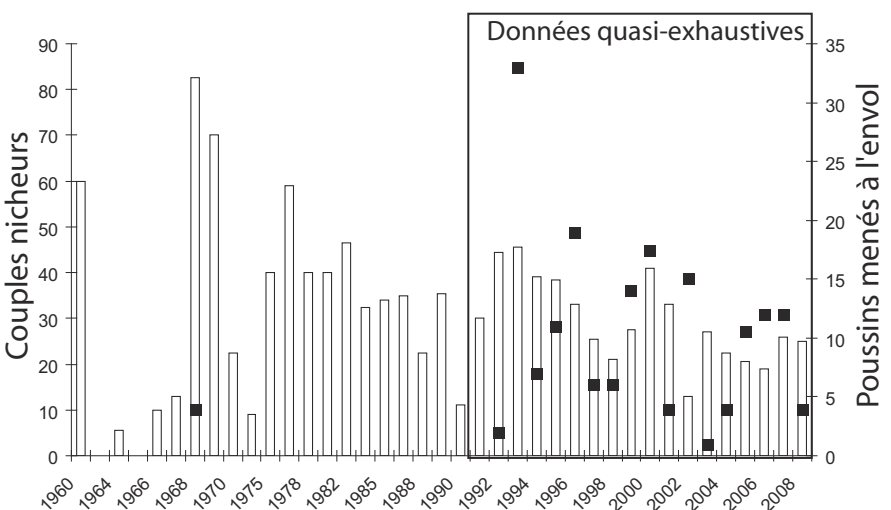

- Poussins menés à l'envol

$\square$ Couples nicheurs

annuelle des colonies est de 9,32 couples (écart-type =3,47). Durant cette même période, aucune tendance significative ne se dégage du nombre de poussins menés à l'envol et du succès de reproduction moyen des couples. Les années produisant beaucoup de poussins à l'envol succèdent aléatoirement à celles n'en produisant aucun et le succès moyen de reproduction des couples est de 0,38 poussin mené à l'envol par couple.

\section{RÉPARTITION SPATIALE DES COUPLES}

L'espèce a toujours été cantonnée à la partie la plus occidentale du Marais poitevin, sur une petite dizaine de communes et un total de 36 sites, avec une concentration des couples autour des trois communes de Champagné-les-Marais, Triaize et Saint-Denis-du-Payré (fig. 2 - planche I). Les prés communaux de cette dernière commune, classés en Réserve Naturelle depuis 1976, constituent un des bastions de l'espèce au moins depuis le début du $\mathrm{xx}^{\mathrm{e}}$ siècle : l'espèce y est notée nicheuse au moins à six reprises de 1907 à 1975 (fig. 2) et a accueilli en moyenne plus de 20 couples par an de 1977 à 1991 et 8 couples par an de 1992 à 2008 (fig. 2). De 1907 à 1975, même si les données sont insuffisantes pour estimer la taille de la population annuelle, les prairies de Triaize et de Champagné-les-Marais semblent être les plus attractives avec des sites occupés au moins trois années (fig. 2). À partir de la fin des années 1970 des couples commencent à s’installer plus à l'ouest sur les communes de Lairoux, Curzon et Saint-Benoist-sur-Mer (fig. 2 - planche I). Mais ce n'est véritablement qu'à partir du début des années 1990 que des couples s'installent à l'ouest du Lay, principalement sur la commune d'Angles et, dans une moindre mesure, sur celles de Saint-Benoist-sur-Mer et Longeville-sur-Mer (fig. 2 - planche I).

\section{EFFETS DES MESURES DE GESTION ESPÈCE-CENTRÉE}

Les sites ayant bénéficié d'une pose de radeaux-nichoirs accueillent significativement plus de couples que ceux sans radeaux (3,92 couples contre 1,48 couples; modèle mixte d'analyse de variance $\mathrm{p}=0,002$ ) mais pas significativement plus de poussins (tableau 2). Les sites d'où le bétail a été exclu accueillent à la fois significativement plus de couples (4,41 couples contre $1,19$ couples, modèle mixte d'analyse de variance $\mathrm{p}=2 \mathrm{e}-16)$ et de poussins $(1,37$ poussins contre 0,30 poussin, modèle mixte d'analyse de variance $\mathrm{p}=0,006$ ) que les sites avec bétail (tableau 2). Le succès de reproduction moyen des couples ne diffère en revanche pas en fonction de la pose de radeau ou de l'exclusion du bétail. 
Alain Thomas, Laurent Godet

\begin{tabular}{|c|c|c|c|c|}
\hline \multicolumn{5}{|c|}{ Nombre de couples } \\
\hline & Estimation & Erreur-type & Valeur z & $\operatorname{Pr}(>|z|)$ \\
\hline Ordonnée à l'origine & $-0,6180$ & 0,3031 & $-2,039$ & 0,04145 \\
\hline Facteur « radeau» & $-0,7280$ & 0,2342 & $-3,108$ & 0,00188 \\
\hline $\begin{array}{l}\text { Facteur « exclusion } \\
\text { du bétail » }\end{array}$ & 1,8795 & 0,1807 & 10,403 & $<2 \mathrm{e}-16$ \\
\hline \multicolumn{5}{|c|}{ Nombre de poussins } \\
\hline & Estimation & Erreur-type & Valeur z & $\operatorname{Pr}(>|z|)$ \\
\hline Ordonnée à l'origine & $-2,5271$ & 0,4995 & $-5,059$ & $42,1 \mathrm{e}-07$ \\
\hline Facteur « radeau» & $-0,2250$ & 0,4135 & $-0,544$ & 0,58634 \\
\hline $\begin{array}{l}\text { Facteur « exclusion } \\
\text { du bétail » }\end{array}$ & 0,9022 & 0,3260 & 2,767 & 0,00565 \\
\hline
\end{tabular}

Tableau 2 : Nombre de couples et de poussins en fonction des facteurs « radeaux » et « exclusion du bétail » exclusion» Number of breeding pairs and chicks according to the factors "floating nest platforms » and «cattle

\section{EFFETS DES MESURES DE GESTION CENTRÉE SUR LES MILIEUX}

En moyenne, sur la période 1992-2008, les couples et les poussins ne se concentrent pas significativement plus sur un des trois types de protection. De même, le succès de reproduction moyen des couples ne diffère pas significativement selon le niveau de protection des sites.

En revanche, sur cette même période, les couples tendent à se concentrer de plus en plus sur les sites bénéficiant d'une protection de niveau P2 (valeur « $y$ » du nombre de couples en fonction du temps : GLM, données log transformées $\left.\mathrm{R}^{2}=0,57, \mathrm{p}=0,03\right)$ et c'est également sur ce type de protection que se concentre la production de poussins (valeur « $y$ » du nombre de poussins en fonction du temps : régression linéaire simple, données log transformées $R^{2}=0,37, p=0,01$ ). En revanche, le succès de reproduction moyen des couples ne tend pas significativement à une hausse ou une baisse selon les trois niveaux de protection

\section{Discussion}

\section{UNE POPULATION RÉDUITE ET EN DÉCLIN AU SEIN DE « MILIEUX DE SUBSTITUTION »?}

A l'image de la situation néerlandaise, où toutes les populations de Guifette noires en déclin ont des succès de reproduction faibles (van der Winden, 2005), la population en déclin du Marais poitevin a des succès moyens de reproduction particulièrement faibles avec 0,38 poussin par couple de 1992 à 2008. Les abandons, réinstallations et échecs des nidifications sont particulièrement fréquents. Pour la période 1992-2008, les abandons partiels ou totaux des colonies ont concerné près de $70 \%$ des installations (obs. pers.). Pour près de la moitié des cas, la cause d'échec n'a pas pu être déterminée, mais les assèchements prématurés ou des niveaux d'eau trop faibles sont à l'origine de $28 \%$ des causes d'échec, suivis par les destructions de pontes par le bétail (11\%), l'action des Cygnes tuberculés $(9 \%)$ et les prédations naturelles (7\%) (obs. pers.). Ce fort taux d'échec des nidifications se situe dans la fourchette haute de ce qui est observé habituellement dans les colonies de Guifettes noires, avec 29 à $96 \%$ de taux de succès de nidification (Servello, 2000). En Europe, comme en Amérique du Nord, les causes d'échecs sont principalement liées à l'inondation des nids suite à de fortes pluies (Bergman et al., 1970; Dunn, 1979; Macikunas, 1993; Mazzocchi et al., 1997; Gilbert et Servello, 2005) et la mortalité des poussins est en grande partie liée à leur prédation (Maxson et al., 2007; Heat et Servello, 2008) et parfois à un manque de ressource trophique (Beintema, 1997). Aux Pays-Bas, J. van der Winden (2005) liste trois prin- 
cipaux facteurs d'échec de reproduction : le manque de substrats adéquats pour la construction de nids, la perturbation des colonies et le manque de ressource trophique pour les jeunes. Les facteurs humains sont liés bien évidemment à l'empiètement d'espaces artificialisés sur les surfaces en eau et la végétation aquatique (Novak, 1992).

La qualité générale des milieux est probablement la cause première de ces forts taux d'échecs observés en Marais poitevin. Les processus anciens d'artificialisation de ce marais, qui se sont poursuivis ces dernières décennies, réduisent significativement les espaces favorables aux Guifettes noires et leur fonctionnalité. Aujourd'hui, il n'existe plus dans le Marais poitevin d'espace de marais naturel, ce qui nous permet de qualifier cette zone humide d'agrosystème. L'espèce est dès lors contrainte de se cantonner aux prairies pâturées les plus humides, à tel point qu'aujourd'hui le système pastoral en prairie humide et la nidification de la Guifette noire est présenté comme un tandem indissociable. Cette association entre espèce paludicole patrimoniale et système pastoral a également été mise en avant pour d'autres espèces : Chevalier gambette Tringa totanus ou encore Vanneau huppé Vanellus vanellus (LPO, 2006) et les mesures de conservation les concernant ne correspondent souvent qu'aux modalités de pâturage à privilégier (par exemple, Durant et al., 2008). Ce postulat oublie le fait que la prairie pâturée peut ne pas être un milieu optimal mais plutôt un milieu de substitution pour ces espèces et particulièrement pour la Guifette noire.

La comparaison des habitats de nidification du Marais poitevin avec ceux d'autres sites européens ou américains confirme l'hypothèse de la prairie pâturée comme milieu de substitution. En Amérique du Nord, les milieux sont d'abord plus vastes et les hauteurs d'eau y sont supérieures : si dans le Marais poitevin les niveaux d'eau oscillent entre 0 et $0,30 \mathrm{~m}$, (obs. pers.), ceux des sites d'Amérique du Nord sont compris entre 0,24 m et 1,31 m (Gilbert et Servello, 2005), entre 0,49 m et 0,59 m (Mazzocchi et al., 1997) ou entre 0,40 m et 0,60 m (Hickey et Malecki, 1997). Généralement, il s'agit de sites combinant à proportion plus ou moins égale surfaces en eau libre et surface avec végétation (Novak, 1992; Hickey et Malecki, 1997). Les groupements végétaux sur lesquels niche la guifette noire en Marais poitevin correspondent principalement aux groupements hygrophiles à Eleocharis spp. et Glyceria fluitans (associations de l'Eleocharito palustris, Oenanthetum fistulosae [de Foucault 1984], Ranunculo ophioglossifolii, Oenanthetum fistulosae [de Foucault 1984]) (obs. pers.), contre des groupements à scirpaies, cariçaies et à Typha associées à des groupements de plantes hydrophytes en Amérique du Nord (Hickey et Malecki, 1997; Gilbert et Servello, 2005 ; Maxson et al., 2007 ; Mazzocchi et al., 1997). Les milieux américains sont des marais de rives d'étangs, de lacs, de bras morts de rivières, parfois avec une gestion hydraulique, mais ce ne sont pas des espaces agricoles (Hickey et Malecki, 1997).

À l'échelle de la France, les principaux sites de nidification sont la Grande Brière et le Lac de Grand-Lieu (Trotignon, 2009). Les zones de nidification y sont plus vastes et sont soumises à des exploitations pastorales plus extensives qu'en Marais poitevin. Les marais de Grand Lieu connaissent également des niveaux d'eau plus élevés et des zones de nidification en partie sans exploitation agricole. En outre, dans le Marais poitevin, le milieu de substitution qu'est la prairie pâturée humide est lui-même en régression. Entre 1973 et 2006, les prairies naturelles ont connu une régression de leur surface de plus de $50 \%$, avec aujourd'hui environ 28000 ha de prairies permanentes (PIMP, données non publiées, fig. 3). Celles-ci sont aujourd'hui principalement restreintes à la partie la plus occidentale du marais, où se cantonnent les guifettes. La Guifette noire est connue pour sa capacité à changer régulièrement de sites de nidification en réponse aux variations environnementales (Dunn, 1979). Dans les cas d'échecs de reproduction des couples, il n'y a donc pas d'autres types de milieux de report dans le Marais poitevin.

Dans ce contexte agricole, et face à une baisse des effectifs de guifettes, les gestionnaires ont opté pour des mesures de gestion de type interventionniste : création d'aires protégées et aménagements pour palier le manque de sites naturels de ponte. Mais s'agit-il de solutions pertinentes à long terme? 

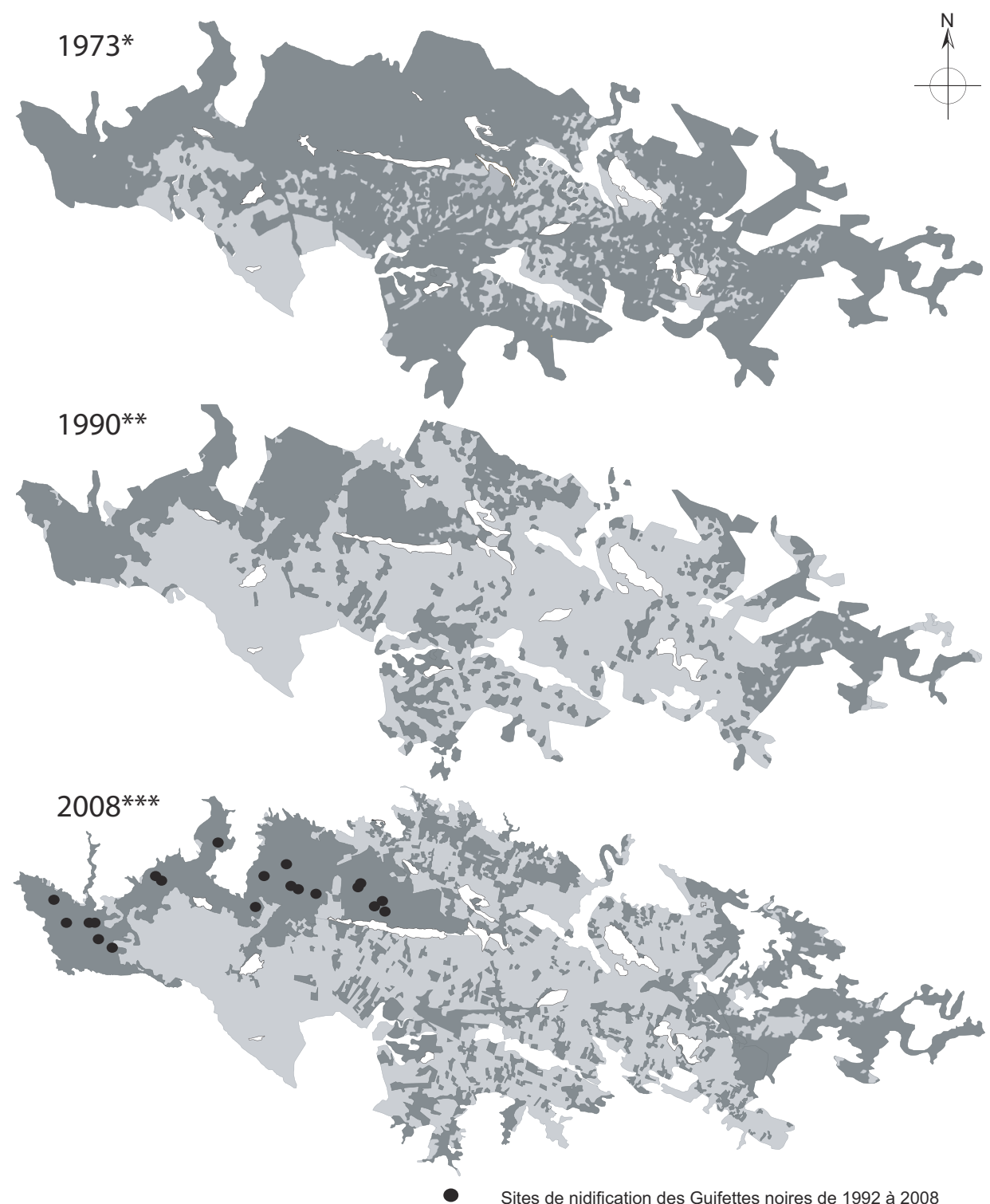

- Sites de nidification des Guifettes noires de 1992 à 2008

Prairies permanentes $(70 \%$ en 2008$)$ et temporaires ( $30 \%$ en 2008$)$

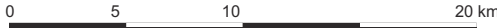

Cultures

Ilôts calcaires

* Modifié d'après IGN, Mission d'étude pour le PNR - 1973

** Modifié d'après étude cartographique par satellite, Ministère de l'Environnement/SATEC - 1990

*** Modifié d'après cartographie du Syndicat Mixte du PIMP - mars 2009 (données non publiées)

Figure 3 : Évolution de l'occupation du sol dans le Marais poitevin de 1973 à 2008

Land-use change in the Marais poitevin from 1973 to 2008 


\section{ESPACES PROTÉGÉS ET MESURES DE GESTION : NÉCESSAIRES MAIS INSUFFISANTS?}

De prime abord, la concentration de plus en plus marquée des couples et des poussins sur les prés communaux bénéficiant d'une gestion environnementale montre l'importance de la gestion environnementale de prairies pour le maintien de la population de guifettes. En revanche, les succès de reproduction des couples ne sont pas meilleurs au sein des espaces protégés $(\mathrm{P} 1)$ ou à gestion environnementale (P2). Nos résultats suggèrent ensuite que la pose de radeaux et l'exclusion du bétail ont un effet positif pour les colonies de guifettes : plus de couples s'installent sur les sites bénéficiant d'une pose de radeaux et plus de couples et de poussins sont produits sur ceux d'où le bétail a été exclu. Ces mesures de gestion tendent donc probablement à attirer significativement les oiseaux sur ces «prairies gérées pour les guifettes». Mais ceci est à nuancer car dans le Marais poitevin ces aménagements sont principalement mis en place avant l'arrivée des oiseaux de retour de leur hivernage, sur des sites a priori les plus favorables à l'espèce. En conséquence, c'est aussi la capacité des gestionnaires à mettre en place des aménagements sur les sites les plus adéquats qui est testée. De plus, dans l'état actuel des connaissances, l'intérêt de ces mesures est à relativiser car le succès moyen de reproduction sur les sites bénéficiant de ces gestions n'est en moyenne pas supérieur. Toutefois, pour tester de manière fine l'efficacité de la pose de radeaux et de l'exclusion du bétail des données supplémentaires, couple par couple, et non pas seulement à l'échelle des colonies, seraient nécessaires. Pour l'instant, la configuration des sites de nidification du marais ne nous a pas permis de suivre individuellement la nidification de chaque couple sous peine d'un dérangement trop important des colonies.

Plusieurs auteurs ont déjà testé l'impact des radeaux sur la nidification des guifettes. D'une manière générale, plus les milieux sont défavorables à l'accueil de l'espèce, plus les couples sélectionnent les radeaux (Rabenold, 1988; Hands et al., 1989, Shealer et al., 2006). Cette installation préférentielle des couples sur sites avec radeaux et leur concentration sur les sites protégés est probablement indicatrice de l'incapacité croissante des milieux contemporains du Marais poitevin à accueillir naturellement l'espèce. Nous estimons que si le déclin de la Guifette noire dans le Marais poitevin peut être ralenti par ces mesures de protection et de gestion des prairies humides, celles-ci, sous leur forme actuelle, ne sont assurément pas suffisantes puisqu'elles ne semblent pas améliorer le succès moyen de reproduction et que la population continue à décliner.

\section{QUELLES SOLUTIONS POUR UNE CONSERVATION DURABLE?}

Les solutions de conservation de la population de guifettes se situent probablement au-delà de mesures techniques. L'accumulation de telles mesures risque d'aboutir à une gestion espècecentrée extrême et non durable. Aux Pays-Bas, les poses de radeaux-nichoirs ont été les premières mesures de gestion mises en place à large échelle pour conserver l'espèce et aujourd'hui ce sont plus de $80 \%$ des couples de Guifettes noires de ce pays qui nichent sur ces aménagements (van der Winden, 2005). Toutefois cette gestion interventionniste espèce-centrée est couplée depuis plusieurs années à une restauration de la fonctionnalité des milieux : politiques de gestion de l'eau, gestion adaptée de la végétation rivulaire et création de petites zones humides propices au développement d'espèces consommées par les guifettes (van der Winden, 2005).

En Marais poitevin, si les actions de protection et de gestion ont probablement ralenti quelque peu le déclin de la population, son maintien peut s'expliquer également par deux facteurs. Tout d'abord, l'espèce est longévive (jusqu'à 17 ans - Cramp, 1985) ce qui explique une certaine inertie des variations d'effectifs face à des perturbations. Ensuite, l'espèce montre une certaine fidélité aux sites de nidification. Depuis le début du $\mathrm{xx}^{\mathrm{e}}$ siècle, elle a toujours été cantonnée à la même partie nord-ouest du Marais poitevin, et, à l'échelle des prairies, les adultes tendent à réutiliser les prairies où des jeunes ont été menés à l'envol l'année d'avant.

Jusqu'à présent, les actions de protection n’ont probablement pas suffisamment été étayées sur une bonne connaissance de l'écologie de la Guifette noire dans le Marais poitevin. Les actions 
Alain Thomas, Laurent Godet

menées, très techniques, s'apparentent à une gestion interventionniste similaire dans l'esprit à l'aménagement et à la gestion agricole du Marais poitevin. La naturalité des prairies humides est aussi bien souvent surestimée, assimilant des milieux agricoles à des milieux naturels. Il en découle une mauvaise appréciation du potentiel d'accueil de la zone humide pour des espèces comme la Guifette noire qui sont inféodées aux marais très inondés. Dans une région aussi artificialisée que l'est le Marais poitevin, la protection de la Guifette noire s'est donc organisée au sein d'une vision aménagiste d'un marais façonné par l'Homme. Cette vision anthropocentrée de la nature est probablement en partie la cause de l'échec des actions de protection.

Il serait opportun d'ouvrir la réflexion sur la conservation de la Guifette noire dans le Marais poitevin à la fonctionnalité des milieux. Une orientation de la gestion de certains espaces vers des milieux plus humides à végétation de scirpaie et de magnocariçaie pourrait offrir aux guifettes des habitats plus en rapport avec leurs exigences écologiques. Dans l'idéal, des groupements végétaux de plusieurs dizaines d'hectares, en relation avec des espaces riches en alimentation et des zones d'eau libre vastes, seraient véritablement en mesure de permettre la nidification de l'espèce, ceci ne venant pas en opposition avec la préservation des prairies humides mais en complément. Reste à savoir ci cela est culturellement et techniquement possible au sein d'un espace essentiellement voué à l'agriculture.

\section{Conclusion}

La petite population de Guifettes noires du Marais poitevin (une trentaine de couples, soit le dixième des effectifs nationaux) est en déclin significatif au moins depuis quarante ans. Les mesures de gestion centrées sur l'espèce, semblent a priori favorables à l'installation de couples reproducteurs, mais ne parviennent ni à augmenter leur succès reproducteur ni à enrayer le déclin de la population. Les sites de nidification de l'espèce sont aujourd'hui localisés exclusivement sur les prairies humides, en pleine régression à l'échelle du marais, et se concentrent de plus en plus sur celles bénéficiant d'une gestion environnementale. A l'image d'autres espèces telles que le Vanneau huppé ou le Chevalier gambette, la Guifette noire est souvent assimilée à une espèce typique de la prairie pâturée, parfois qualifiée d'espèce « anthropophile » car associée aux activités humaines. Il convient sans doute aujourd'hui de reconsidérer ce caractère « anthropophile » non pas comme une relation symbiotique Homme-Nature de type "gagnant-gagnant », mais plutôt comme une adaptation d'espèces de milieux humides à des milieux de substitution fortement modifiés par les activités humaines. Le manque d'efficacité générale des mesures de conservation de ces espèces devrait élargir les perspectives de conservation vers une restauration de la fonctionnalité de milieux humides à large échelle, éventuellement laissés en évolution libre, plutôt que l'accumulation de mesures ponctuelles interventionnistes. Le passage progressif d'une gestion conservatoire interventionniste à une non-intervention (Schnitzler et al., 2008; Génot, 2008) reste cependant un processus lent et très récent et requiert une relation plus humble et moins prométhéiste de la conservation de la Nature. 


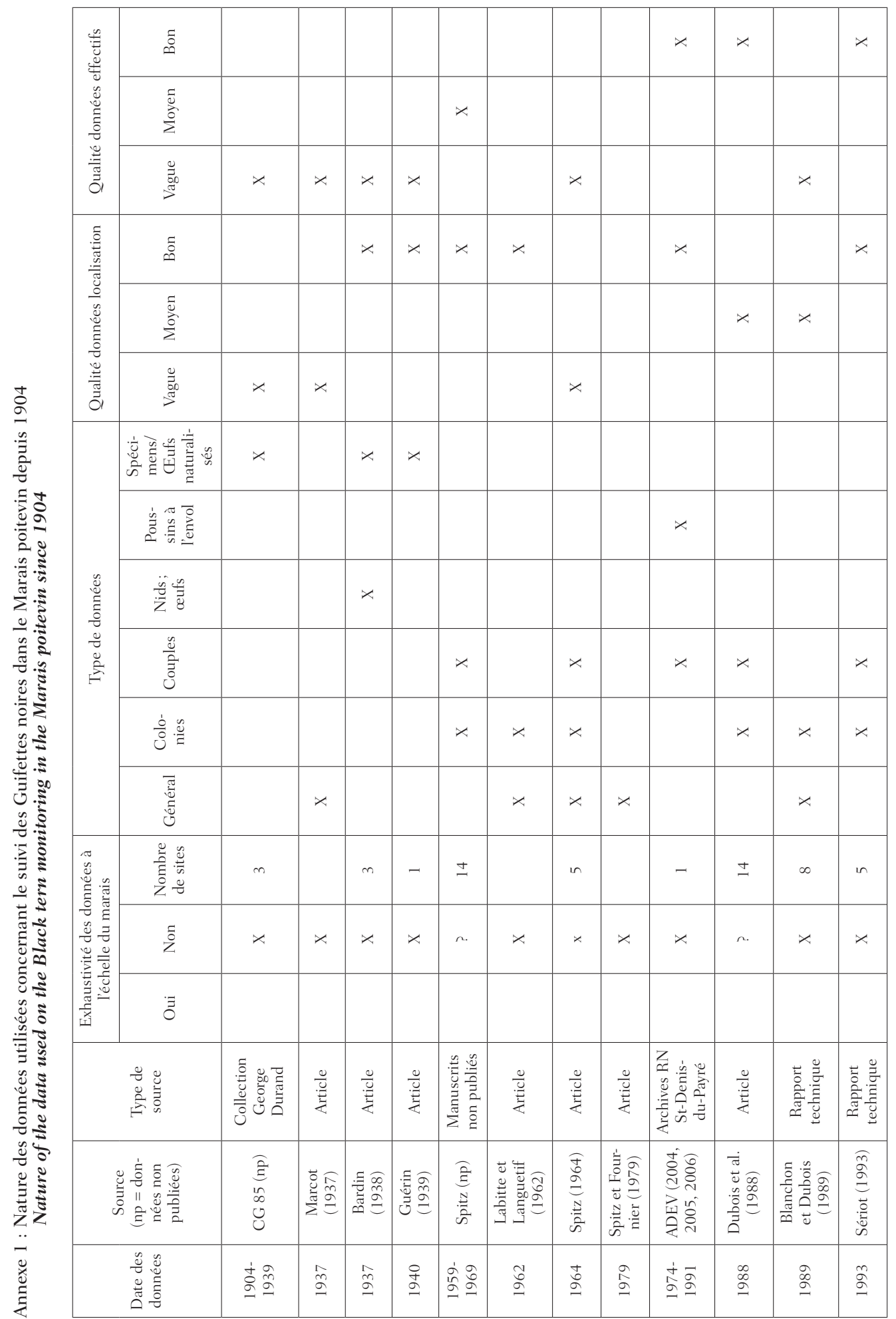


20

Alain Thomas, Laurent Godet

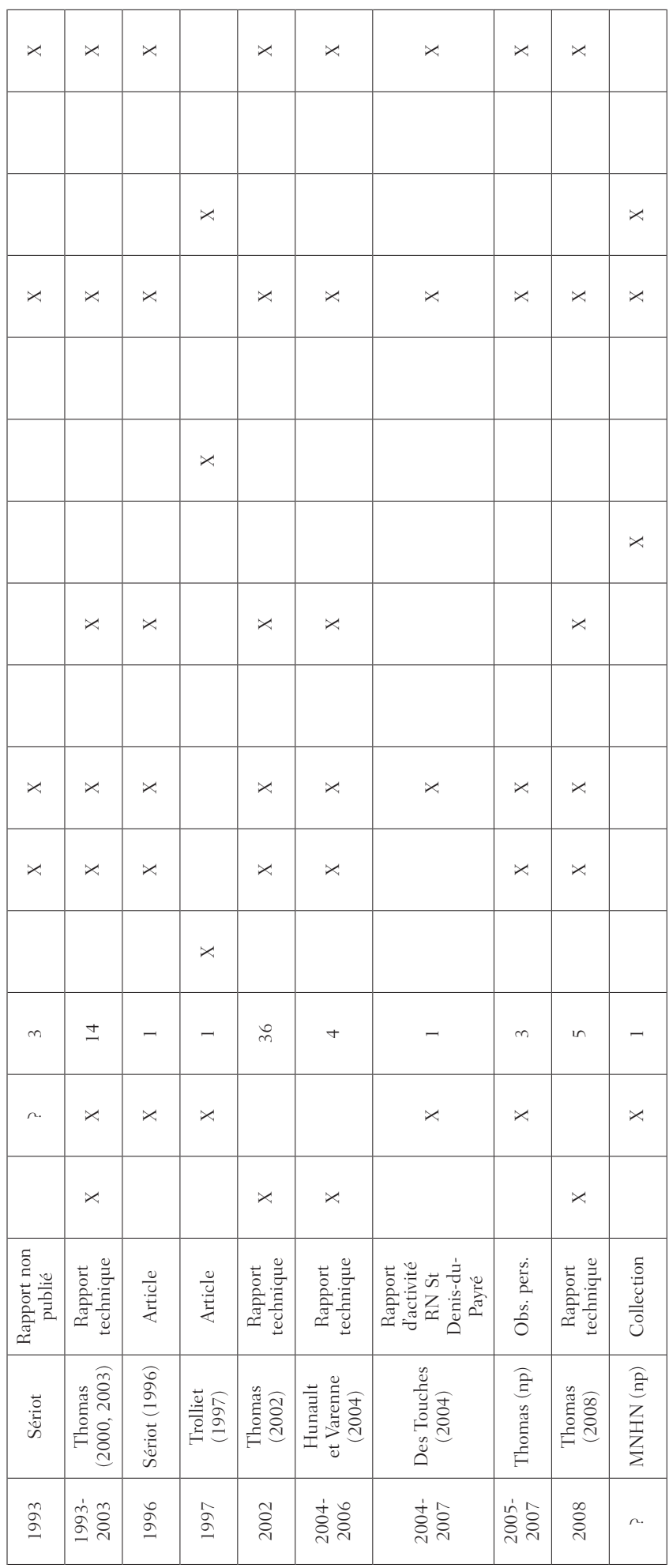




\section{Bibliographie}

ADEV, 2004. Réserve Naturelle de Saint Denis-du-Payré. Résumé du Plan de Gestion 2004-2010.

ADEV, 2005. Suivi annuel et action de protection de la Guifette noire (Chlidonias niger) en Marais Poitevin 2005, Rapport d'étude LIFE, ADEV - Parc Interrégional du Marais Poitevin.

ADEV, 2006. Suivi annuel et action de protection de la Guifette noire (Chlidonias niger) en Marais Poitevin 2006, Rapport d'étude LIFE, ADEV - Parc Interrégional du Marais Poitevin.

Bardin M., 1938. Premières notes sur le marais vendéen, L'Oiseau et la Revue Française d'Ornithologie, vol. 8, p. $78-83$.

Beintema A. J., 1997. European Black Terns (Chlidonias niger) in Trouble : Examples of Dietary Problems, Colonial Waterbirds, vol. 20, p. 558-565.

Bergman R. D., Swain P. S., Weller M. W., 1970. A comparative study of nesting Forster's and Black Terns, Wilson Bulletin, vol. 82, p. 435-444.

Blanchon J.-J., Dubois P. J., 1989. Mise au point de techniques de valorisation rationnelle et judicieuse des espaces naturels du Marais poitevin. Importance des zones humides Baie de l'Aiguillon et marais communaux pour l'avifaune, Rapport d'étude, LPO/ministère de l'Environnement/Direction de la Protection de la Nature.

Brinson M. M., Malvarez A. I., 2002. Temperate freswater wetlands: types, status, and threats, Environmental Conservation, vol. 29, p. 115-133.

Cramp S., 1985. Handbook of the birds of Europe, the Middle East, and North Africa. Vol. 4: terns to woodpeckers, Oxford University Press, New York.

Dahl T. E., 2000. Status and trends of wetlands in the conterminous United States, 1986-1997, U.S. Department of Interior, Fish and Wildlife Services. Washington, DC, US.

Des Touches H., 2004. Réserve Naturelle «Michel Brosselin » St Denis-du-Payré (Vendée), Rapport d'activité septembre 2003-Août 2004.

Dubois P. J., Fouguet M., Yesou P., 1988. La nidification des Laridés entre Loire et Gironde, Alauda, vol. 56, p. 2-34 et 413-428.

Dunn E. H., 1979. Nesting biology and development of young in Ontario Black Terns, Canadian FieldNaturalist, vol. 93, p. 276-281.

Durant D., Tichit M., Fritz H., Kerneïs E., 2008. Field occupancy by breeding lapwings Vanellus vanellus and redshanks Tringa totanus in agricultural wet grasslands, Agriculture, Ecosystems and Environment, vol. 128, p. 146-150.

Finlayson C. M., Davidson N. C., 1999. Global review of wetland resources and priorities for wetland inventory: Summary report, dans finlayson C. M., Spiens A. G. (dir.), Global Review of Wetland Resources and Priorities for Wetland Inventory, CDROM, Canberra, Australia, Supervising Scientist Report 144.

GÉnot J.-C., 2008. La nature malade de la gestion, Paris, Sang de la Terre, 239 p.

Gilbert A. T., Servello F. A., 2005. Water Level Dynamics in Wetland and Nesting Success of Black Tern in Maine, Waterbirds, vol. 28, p. 181-187.

Guerin G., 1939. Ornithologie du Bas Poitou. Les Oiseaux dans la Vendée et quelques cantons limitrophes, L'oiseau et la revue française d'ornithologie, vol. 9, p. 233.

Hands H. M., Drobney R. D., Ryan M. R., 1989. Status of the Black Tern in the northcentral United States, U.S. Fish and Wildlife Service, St. Paul, MN.

Heath S. R., Servello F. A., 2008. Effects of predation and food provisioning on Black tern chick survival, The Wilson Journal of Ornithology, vol. 120, p. 167-175.

Hickey J. M., Malecki R. A., 1997. Nest Site Selection of the Black Tern in Western New York. Colonial Waterbirds, vol. 20, p. 582-595.

Hunault S., Varenne F., 2004. Guifette noire Chlidonias niger. Bilan de la nidification en Marais poitevin année 2004, Rapport d'étude LIFE, ADEV - Parc Interrégional du Marais Poitevin.

Keddy P. A., 2000. Wetland Ecology: Principles and Conservation, Cambridge University Press. 
Alain Thomas, Laurent Godet

Keddy P. A., Fraser L. H., Solomeshch A. L., Junk W. J., Campbell D. R., Arroyo M. T. K., Alho C. J. R., 2009. Wet and Wonderful: the world's largest wetlands are conservation priorities, Bioscience, vol. 59, p. 39-51.

Labitte A., Languetif A., 1962. Notes sur les oiseaux nicheurs du marais vendéen (au printemps 1960), L'oiseau et la revue française d'ornithologie, vol.32, p. 58.

LPO, 2006. Limicoles nicheurs du marais poitevin. Synthèse de l'enquête 2005-2006, Rapport d'étude LPO Parc Interrégional du Marais Poitevin.

Macikunas A., 1993. Hatching success and replacement success of the Black Tern (Chlidonias niger) of the Kaunas Sea, Acta Ornitologica Lituanica, vol. 7-8, p. 107-114.

Мавсот C., 1937. Oiseaux de la Baie de l'Aiguillon-sur-Mer (Vendée) et du marais environnant, Alauda, vol. 9, p. 77.

Maxson S. J., Fieberg J. R., Riggs M. R., 2007. Black Tern Nest Habitat Selection and Factors Affecting Nest Success in Northwestern Minnesota, Waterbirds, vol. 30, p. 1-9.

Mazzocchi I. M., Hickey J. M., Miller R. L., 1997. Productivity and Nesting Habitat Characteristics of the Black Tern in Northem New York, Colonial Waterbirds, vol. 20, p. 596-603.

MEA (Millenium Ecosystem Assessment), 2005. Ecosystems and Human Well-being: Wetlands and Water Synthesis, Washington (DC): World Resources Institute.

Novak P.G., 1992. Black Tern, Chlidonias niger, dans Schneider K. S., Pence D. M. (Dir.), Migratory nongame birds of management concern in the northeast, United States Fish and Wildlife Service, United States Department of the Interior, Newton Corner, Massachusetts, p. 149-169.

Rabenold P.P., 1988. 1988 Survey of Black Terns (Chlidonias niger) breeding in Indiana, Indiana Department of Natural Resources, Indianapolis, IN.

Schnitzler A., Génot J.-C., Wintz M., 2008. Espaces protégés : de la gestion conservatoire vers la nonintervention, Courrier de l'Environnement de l'INRA, vol. 56, p. 29-44.

SÉriot J., 1993. Distribution, déterminisme des stationnements et de l'installation de l'avifaune des communaux et des prairies humides du Marais Poitevin. Rapport d'étude, LPO/Parc Naturel Régional du Marais Poitevin/ Val de Sèvre et Vendée.

SÉriot J., 1996. Protection de la Guifette noire Chlidonias niger dans l'Ouest de la France, Ornithos, vol. 3 , p. 130-134.

Servello F. A., 2000. Population and research priorities for Black Terns developed from modelling analyses, Waterbirds, vol. 23, p. 440-448.

Shealer D. A., Buzzell J. M., Heiar J. P., 2006. Effect of floating nest platforms on the breeding performance of Black Terns, Journal of Field Ornithology, vol. 77, p. 184-194.

Spitz F., 1964. Notes sur l'avifaune nicheuse de la région de Saint-Michel-en-l'Herm (Vendée), L'Oiseau et Revue Française d'Ornithologie, vol. 34.

Spitz F., Fournier O., 1979. Les activités de la station INRA de Saint-Michel-en-l'Herm (Vendée) dans le domaine du gibier d'eau (1959-1969), Bulletin Mensuel de l'Office National de Chasse, Numéro spécial mai 1979, p. 93-108.

Thomas A., 2000. Suivi de la nidification de la Guifette noire (Chlidonias niger) dans le Marais poitevin, saison 2000, Rapport d'étude ADEV, Parc Interrégional du Marais Poitevin, 15 p.

Thomas A., 2002. La Guifette noire (Chlidonias niger) dans le Marais Poitevin, Historique, suivi d'une population et plan de sawvegarde, Rapport d'étude ADEV, Parc Interrégional du Marais Poitevin, 90 p.

Thomas A., 2003. Guifette noire (Chlidonias niger), Bilan de la nidification en Marais poitevin année 2003, Rapport d'étude ADEV, Parc Interrégional du Marais Poitevin, 11 p.

Thomas A., 2008. Suivi de la population reproductrice de Guifettes noires (Chlidonias niger) du Marais poitevin année 2008, Rapport d'étude Alain Thomas, Parc Interrégional du Marais Poitevin, 34 p.

Trolliet B., 1997. Une ponte de Guifette noire Chlidonias niger particulièrement précoce, Nos Oiseaux, vol. 44 , p. 243.

Trotignon J. (Coord.), 2009. Suivi de la nidification des guifettes en France, Groupe Guifettes France, 13 p.

Tucker G. M., Heath M. F., Tomialojc L., Grimmett R. F. A., 1994. Birds in Europe: their conservation status, BirdLife International. 
Van der Winden J., Hagemeijer W., Terlouw R., 1996. Heeft de Zwarte Stern Chlidonias niger een toekomst als broedvogel in Nederland? Limosa, vol. 69, p. 149-164.

VAN DER Winden J., 2005. Black Tern Chlidonias niger conservation in the Netherland - a review, Vogelwelt, vol. 126, p. 187-193.

Cet article a été reçu le 9 novembre 2009 et définitivement accepté le 15 juin 2010. 
planche I (Alain THOMAS, Laurent GODET - La conservation d'une espèce patrimoniale en déclin...)
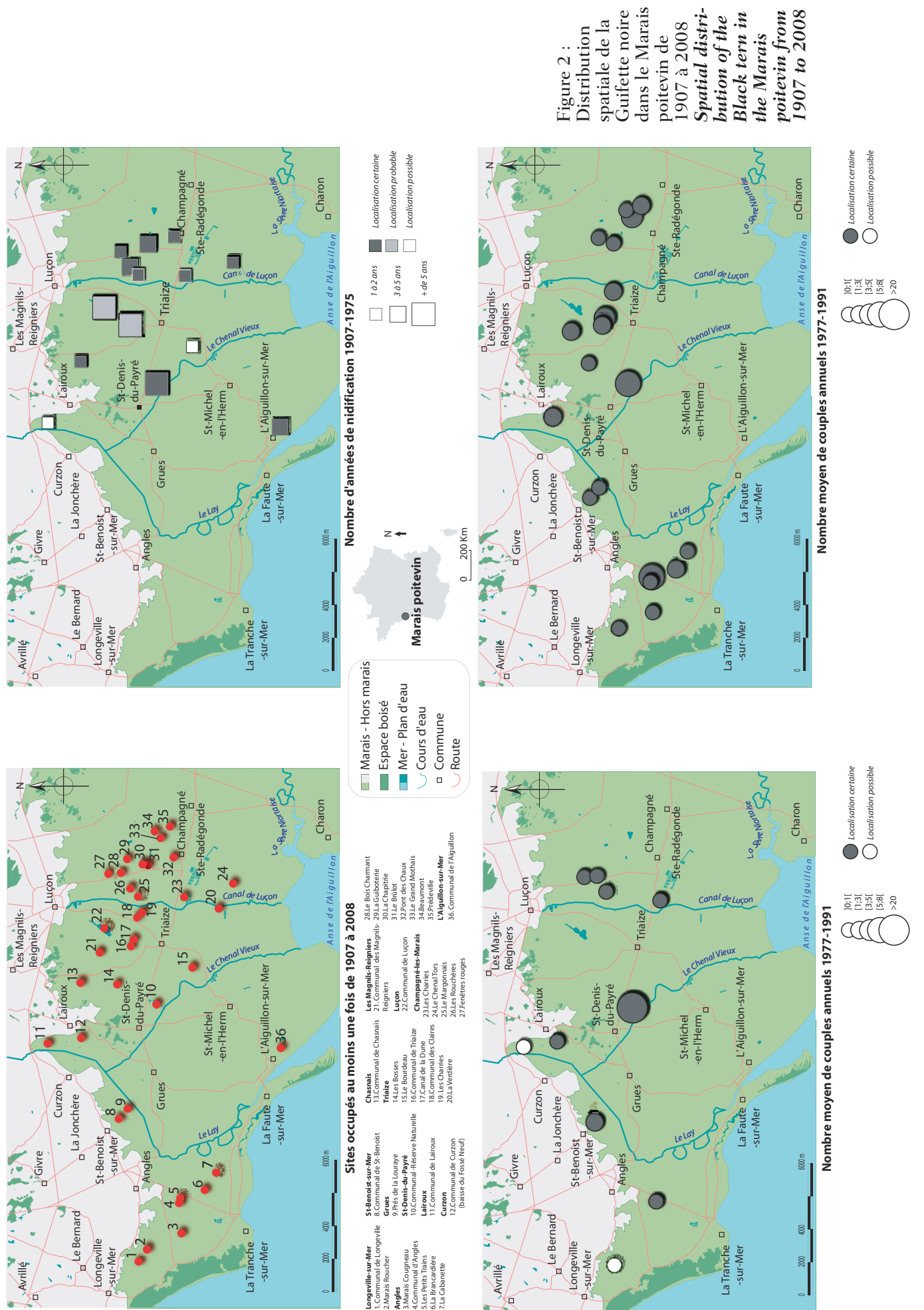

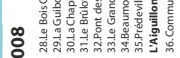

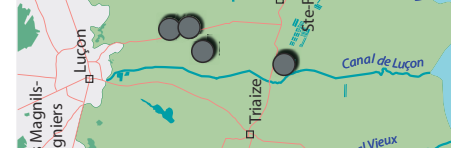

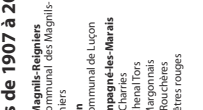
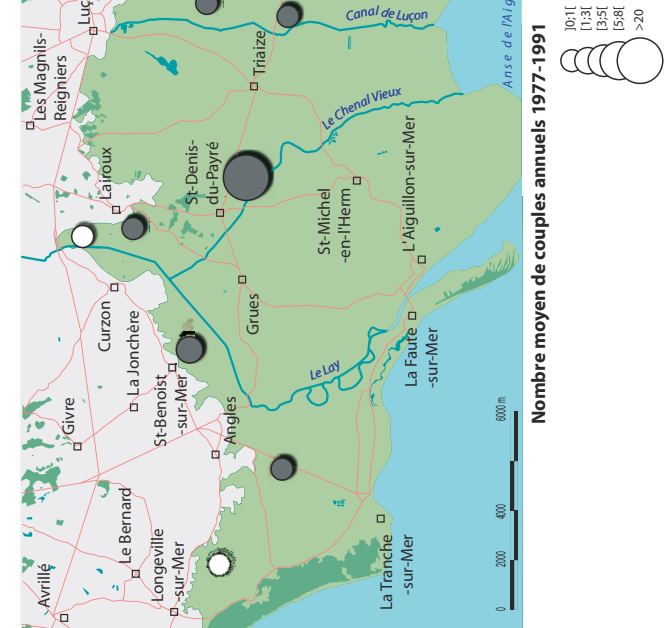